\title{
Effect of Rubber Deformation Along Sliding Contact on Its Stress and Friction Force: A Numerical Investigation
}

\author{
Budi Setiyana ${ }^{1, *}$ Rifky Ismail $^{1}$ Sugiyanto $^{1}$ Jamari Jamari $^{1}$ \\ ${ }^{I}$ Engineering Design and Tribology Laboratory, Mechanical Engineering Department, Faculty of Engineering, \\ University of Diponegoro, Jl. Prof. Soedharto SH, Kampus Tembalang, Semarang 50275 \\ "Corresponding author.Email: bsetiyana@yahoo.com
}

\begin{abstract}
Rubber is a material that easily deforms when subjected to friction, such as in tires, seals and transmission belts. It is very important to understand the relationship of deformation to its stress and friction contact on the rubber. The stress and friction force are closely related to the strength and braking capacity of the rubber, especially for the rubber used in tires. This paper investigates the effect of rubber deformation along friction on its stress and friction force of the rubber surface. Friction contact between the rubber surface and a rigid indenter was modelled numerically using Finite Element (FE) software. Analyzed rubber was Styrene Butadiene Rubber (SBR) and considered as a hyper-elastic material based on Mooney-Rivlin model. Analysis was carried out at specified depth of sliding, surface roughness and sliding speed. Results showed that the degree of deformation will be higher for the larger sliding depth and higher surface roughness. Along friction, the high degree of deformation causes high stress and friction forces of the rubber surface, however, fluctuating value of those parameters is obtained here.
\end{abstract}

Keywords: Deformation, Finite Element, Friction, Rubber.

\section{INTRODUCTION}

Rubber or elastomer is a unique material with compliant, soft, and hyper-elastic behavior $[1,2]$. Consequently, it is easy to deform when subjected to friction contact, such as used in tires, seals, and transmission belts. Due to its properties, it is very important to understand the degree and shape of deformation of the rubber with respect to its stress and friction forces along sliding contact. In sliding contact, the stress of the rubber is strongly related to its performance especially regarding to abrasion resistance. Meanwhile, the friction force that occurs are closely related to the braking capacity of the rubber, especially for the rubber used in tires.

Rubber material was usually modeled as a viscoelastic or hyper-elastic material [1,2]. As a viscoelastic material, hysteresis effect is regarded especially on high damping value. It is commonly found on high compounded rubber. If the damping value is small, hysteresis effect is regardless, and the rubber is often modeled as a hyper-elastic material. There were some formulations for modelling the rubber as a hyper- elastic model that were proposed by several experts. Because of nonlinearity behavior, most of rubber analysis including its deformation was performed using numerical method through Finite Element simulation [3].

Rubber is a consumable part especially when used as a vehicle tire. Friction contact between tire rubber surface and its counter surface is mostly studied. Degree of deformation on the tire rubber strongly depends on the stiffness of tire rubber, load exerted and road condition $[4,5]$. Several models were proposed to analyze this case; however, some simplifications of those models were made so that it is easy to be analyzed. Related to that case, this paper proposed the sliding contact between a flat rubber surface and a rigid counter surface. Rubber material was a vulcanized rubber that is usually used as tire and the counter surface was a rigid blade indenter. Input parameter were depth of indentation and contact surface roughness. Sliding contact simulation was carried out using Finite Element (FE) software package so that the relationship between deformation and stress as well as friction force is obtained. 


\section{METHOD}

The present analysis was carried out using a commercial Finite Element (FE) software numerically, ABAQUS 6.11 [6]. The vulcanized rubber analyzed was SBR-25 (Compounded Styrene Butadiene Rubber) that was compounded by a carbon black of $25 \%$ weight. By

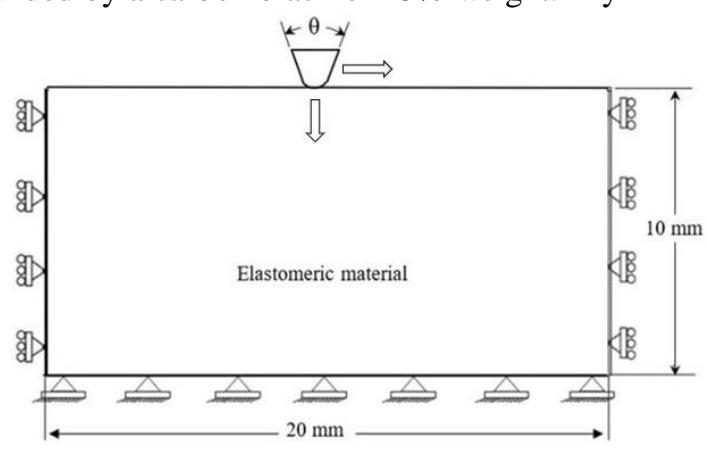

(a) modeling the rubber as a hyper-elastic material, tensile test of rubber specimen was required for input data in FE simulation. The input data was some constants of Strain Energy Function (SEF) term [7]. The required constants data of SEF were taken from Liang's experiment [8] that was obtained by a tensile test up to specified strain level.

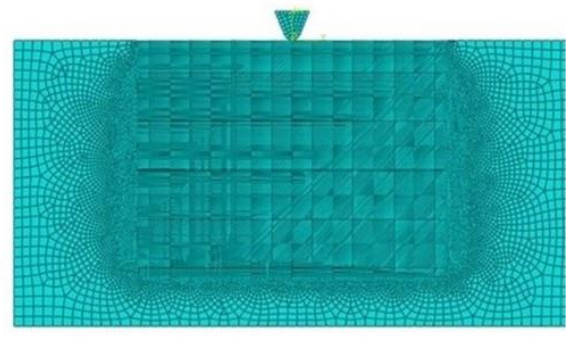

(b)

Figure 1 Schematic illustration of sliding contact (a) Schematic model and (b) FE model.

An illustration of a rigid counter surface sliding on the rubber surface is shown schematically in Figure 1(a). The rigid blade indenter with tip radius of $0.5 \mathrm{~mm}$ slides on the rubber (elastomer) surface. The boundary conditions of the Finite Element model are also depicted schematically. The rubber specimen with $10 \mathrm{~mm}$ high, 20 $\mathrm{mm}$ wide and $10 \mathrm{~mm}$ thick was analyzed in the plane strain type. The FE analysis was performed at a constant sliding velocity of $5 \mathrm{~mm} / \mathrm{sec}$ with maximum sliding displacement of $4.0 \mathrm{~mm}$ in some various input data i.e. depth of sliding indentation and surface roughness. The surface roughness was stated by an adhesion coefficient of friction (adhesion COF). The selected depth of sliding was $0.5,1.0$ and $2.0 \mathrm{~mm}$, moreover the adhesion COF was 0.0 and 0.5 .

Simulation was performed in static as well as sliding contact. Simulation was started by providing a specified depth of sliding (before sliding) that was in static condition, then was continued in sliding condition and finally is stopped in $4 \mathrm{~mm}$ sliding displacement. The FE simulation output was presented in the form of

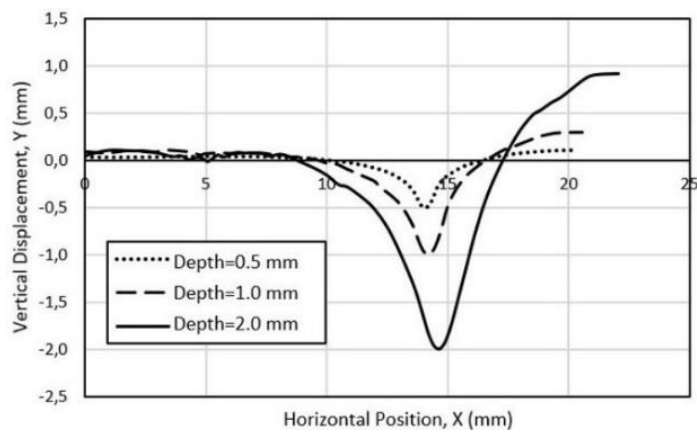

(a) deformation contour and stress pattern. On the other hand, friction force occurring along sliding contact was also investigated. From these results, the stress as well as coefficient of friction can be analyzed and then presented with respect to the various sliding depth and surface roughness.

\section{RESULT AND DISCUSSION}

At first, the contour of rubber surface deformation along sliding contact is depicted in Figure 2. The indenter slides on the rubber surface in the right direction. Figure 2(a) shows the deformation contour on various depth of sliding i. e. $0.5 \mathrm{~mm}, 1.0 \mathrm{~mm}$ and $2.0 \mathrm{~mm}$ with the adhesion COF of 0.5. It is shown that the rubber surface on the right side is higher than the left side, especially on the large depth of sliding. It is also shown that the contour surface in the left side is relatively not so different among those depth of sliding. Thus, in shortly, the larger depth of sliding yields to the higher difference surface contour on the right side or front of sliding direction than the left side.

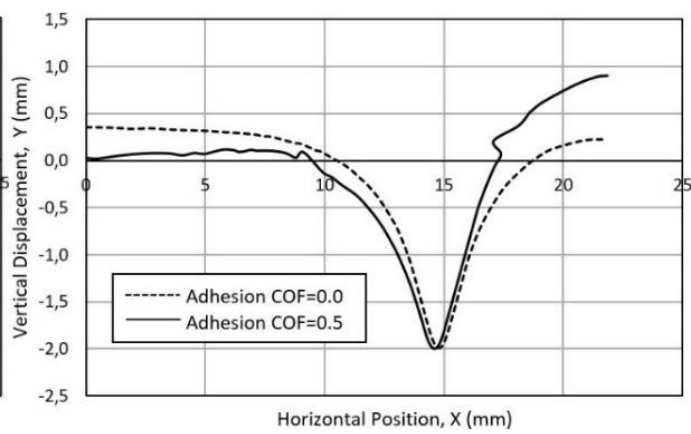

(b)

Figure 2 Contour of rubber surface deformation (a) on various depth of sliding at adhesion COF of 0.5 and (b) on various surface roughness (adhesion COF) at depth of sliding of $2.0 \mathrm{~mm}$. 
The comparison between contour deformation on the smooth and rough surface along sliding in the right direction is depicted in figure 2(b) that represented by the adhesion COF of 0.0 and 0.5 respectively. It is seen that in the smooth surface, there is no practically difference surface contour between the left and right side. It is different to the sliding contact on the rough surface that there is a difference of the contour surface between both sides. Thus, higher surface roughness also provides higher difference of the surface contour between left and right side.

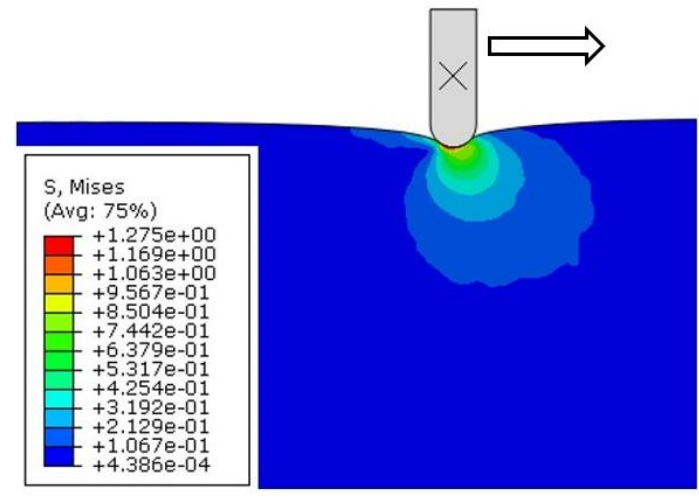

(a)
Von Mises stress pattern on various depth of sliding is depicted in figure 3 with the indenter moving in the right direction. The arrow indicates the direction of sliding. The given depth of sliding was $0.5 \mathrm{~mm}, 1.0 \mathrm{~mm}$ and 2.00 with the given adhesion COF was 0.5 that represents the rough surface condition. It can be seen that the larger depth of sliding provides higher stress intensity. In general, the maximum stress intensity is located below the indenter tip, however the high stress intensity area is located toward to the sliding direction. It agrees with the rubber abrasion phenomena that the crack initiation along abrasion occurred on its location $[5,8]$.

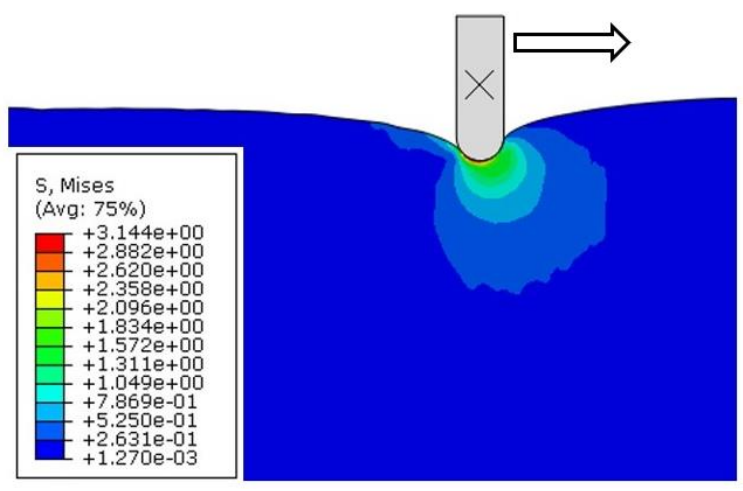

(b)

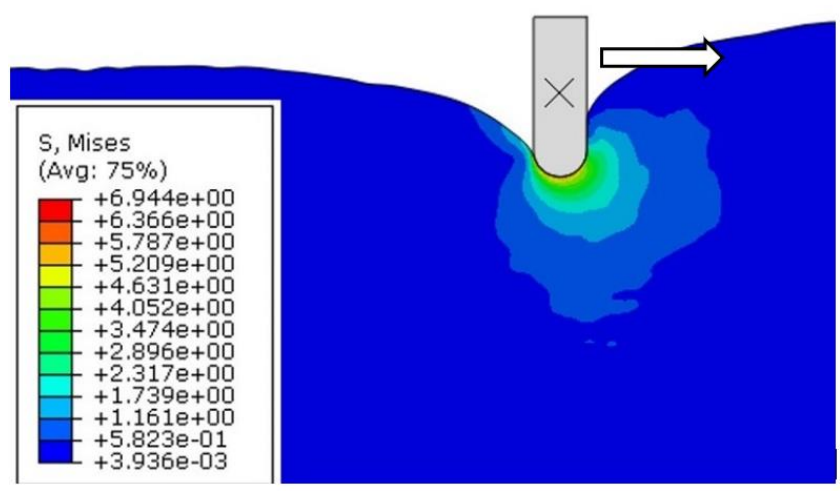

(c)

Figure 3 Stress pattern of the sliding contact with the adhesion COF of 0.5 for the depth of sliding of (a) $0.5 \mathrm{~mm}$, (b) $1.0 \mathrm{~mm}$ and (c) $2.0 \mathrm{~mm}$.

The comparison the von Mises stress pattern on various contact surface roughness is presented in figure 4 with the adhesion COF of 0.0 (represents the smooth surface) and 0.5 (represents the rough surface). In this case, the used indenter is a rigid blade indenter with 45 degrees wedge angle with $0.5 \mathrm{~mm}$ tip radius. It is shown that the stress pattern of the smooth surface in figure 4(a) is close to the symmetrical form. This phenomenon is as in the static indentation case. On the other hand, the stress pattern on the rough surface does not provide in symmetrical form and the large stress area is located toward the sliding direction. In general, the maximum stress intensity occurring in the smooth surface in figure 4(a) is lower than in the rough surface as viewed in figure 4(b). Accordingly, the higher surface roughness also provides the higher stress intensity. 


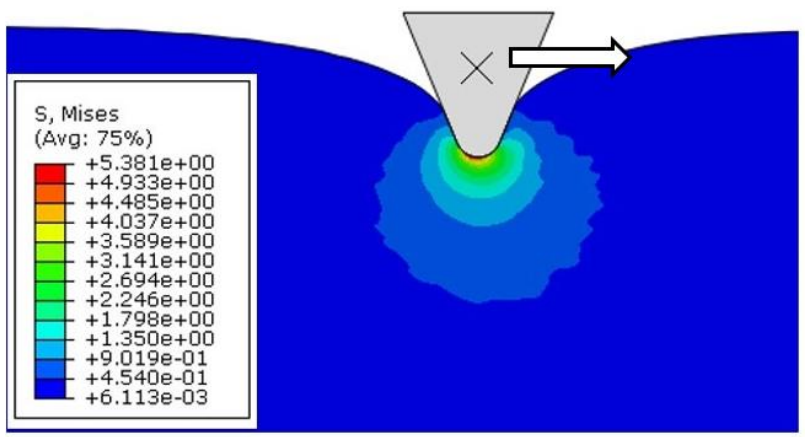

(a)

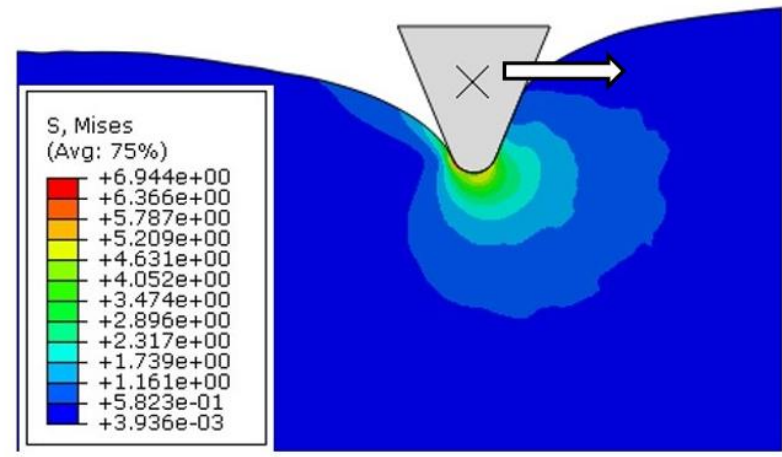

(b)

Figure 4 Stress pattern on various surface roughness using 45 degrees of blade tip indenter in (a) smooth surface and (b) rough surface. The arrow indicates the direction of sliding.

Coefficient of friction (COF) is an important parameter in friction contact that obtained from tangential force divided by normal force along friction. It is strongly related to the braking capacity of the tire-road contact cases. In general, COF consists of the adhesion component due to the contact surface roughness and hysteresis component due to the deformation degree.
High degree of deformation also causes high hysteresis effect as well as friction force. However, COF due to the deformation is difficult to be analyzed theoretically, therefore the numerical method is frequently applied. In COF analysis, the adhesion COF is assumed to be constant for all case of deformation degree, thus the $\mathrm{COF}$ due to deformation is often solely presented in analysis.

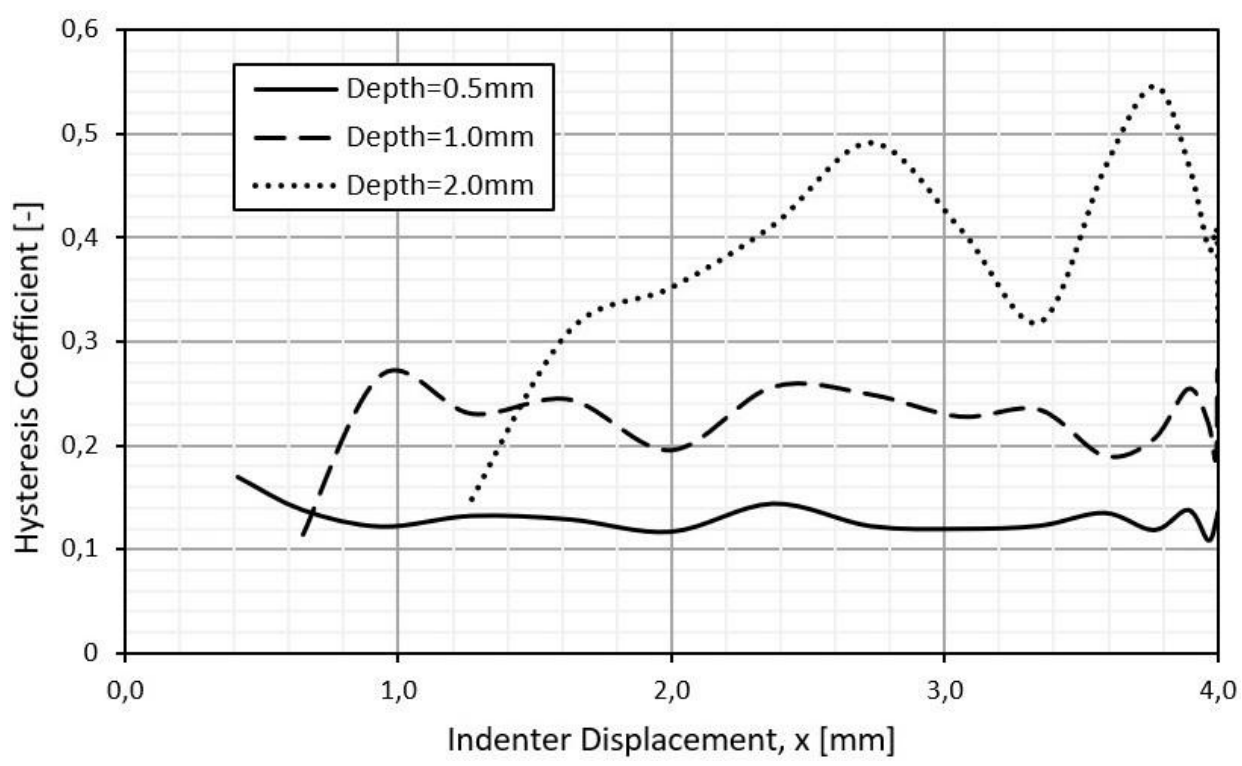

Figure 5 Hysteresis coefficient component on the sliding contact with some various depth of sliding i. e. $0.5 \mathrm{~mm}, 1.0$ $\mathrm{mm}$ and $2.0 \mathrm{~mm}$

In this study, hysteresis coefficient on sliding contact with respect to the sliding displacement is depicted graphically in figure 5 in the various depth of sliding i. e. $0.5 \mathrm{~mm}, 1.0 \mathrm{~mm}$ and $2.0 \mathrm{~mm}$. Simulation was performed using straight blade indenter that slides along $4 \mathrm{~mm}$ on the rubber surface. It is shown that the larger depth of sliding provides larger hysteresis coefficient with fluctuating value. Therefore, it also provides the fluctuating value of the coefficient of friction. This case indicates that large sliding depth provides fluctuation or oscillation of the rubber surface along sliding contact. Shortly, high degree of deformation along sliding provides oscillation or vibration on the rubber surface.

\section{CONCLUSION}

In sliding contact, degree and shape of the rubber surface deformation strongly depends on the depth of sliding and contact surface roughness. The larger sliding depth and higher surface roughness also provide higher degree of deformation, stress intensity and friction force. Stress pattern and contour of deformation along sliding on the smooth surface provides more symmetrical form than that of the rough surface. Regarding the friction force along sliding, the coefficient of friction due to deformation or hysteresis occurs to fluctuate on the 
large depth of sliding indentation. Thus, high degree of deformation along sliding provides oscillation or vibration on the rubber surface.

\section{ACKNOWLEDGMENT}

This study was granted by Engineering Faculty of Diponegoro University, Semarang Indonesia in the scheme of Strategic Research for year of 2020.

\section{REFERENCES}

[1] Gent, A. N. (2013). Rubber Elasticity: Basic Concepts and Behavior. The Science and Technology of Rubber, 1-26. https://doi.org/10.1016/B978-0-12-3945846.00001-7.

[2] Savkoor, A. R. (1986). Mechanics of sliding friction of elastomers. Wear, 113(1), 37-60. https://doi.org/10.1016/0043-1648(86)90055-4.
[3] MSC Software Whitepaper. (2000). Nonlinear finite element analysis of elastomers - technical paper. www.mscsoftware.com.

[4] Setiyana, B., Prabowo, C., Jamari, J., Ismail, R., Sugiyanto, S., \& Saputra, E. (2020). Numerical investigation of the hardness of tire rubber material by indentation method. Journal of Physics: Conference Series, 1517(1). https://doi.org/10.1088/1742-6596/1517/1/012020.

[5] Zhang, S. W. (2004). Tribology of Elastomers: Tribology and Interface Engineering Series. Amsterdam: Elsevier.

[6] ABAQUS 6.11 (2010). Abaqus Analysis User's Manual. 15.

[7] Yeoh, O. H. (1990). Characterisation of Elastic Properties of Carbon Black Filled Rubber Vulcanizates. Rubber chemistry and technology Vol. 63, p. 792-805, 1990.

[8] Liang, H. (2007). Investigating the Mechanism of Elastomer Abrasion. August, 1-192. 